\title{
On Completely YJ-injective Rings
}

Raida D. Mahammod

raida.1961@uomosul.edu.iq
Husam Q. Mohammad

husam_alsabawi@yahoo.com

College of Computer Sciences and Mathematics

University of Mosul, Mosul, Iraq

\section{Received on: 14/02/2011}

\section{ABSTRACT}

Accepted on: 21/06/2011

A ring $\mathrm{R}$ is called completely right YJ-injective (briefly, right CYJ injective ) if every homomorphic image of $\mathrm{R}$ is right $\mathrm{YJ}$-injective. In this paper, we study completely right YJ-injective rings and their connection with Von Neumann regular rings. In addition, we also study regularity of rings whose ring homomorphic images are right YJ-injective as right $\mathrm{R}$-modules

Keywords: Completely YJ-injective Rings, homorphic image, strongly $\pi$-regular ring.

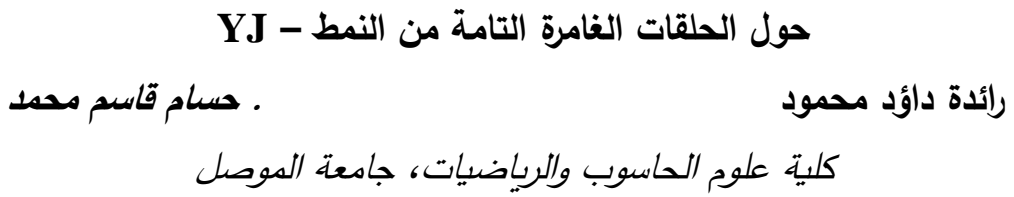

تاريخ قبول البحث: 2011/06/21

تاريخ استلام البحث: 2011/02/14

$$
\begin{aligned}
& \text { الملخص } \\
& \text { يقال للحلقة R أنها حلقة غامرة يمنى تامة من النمط - YJ إذا كان كل صورة تشاكلية لـ R تكون حلقة }
\end{aligned}
$$

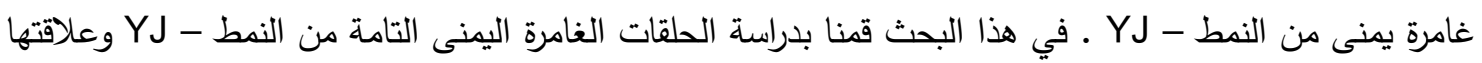

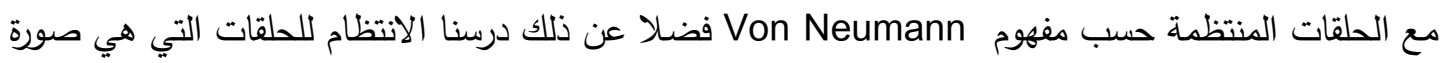

$$
\begin{aligned}
& \text { تشاكلية للحلقات الغامرة اليمنى التامة من النمط - YJ باعتبارها مقاسات يمنى. } \\
& \text { الكلمات المفتاحية: الحلقات الغامرة التامة من النمط - YJ , صورة تثاكلية, الحلقات المنتظمة من النمط - }
\end{aligned}
$$

\section{.1. Introduction:}

Throughout this paper, $\mathrm{R}$ denoted an associative ring with identity, and all modules are unitary. For $a \in R, r(a)$ and $1(a)$ denote the right annihilator and the left annihilator of a, respectively. We write $J(R), Y(R)(Z(R))$ and $N(R)$ for the Jacobson radical, the right (left) singular ideal and the set of nilpotent elements respectively. A right $\mathrm{R}$-module $\mathrm{M}$ is called YJ-injective if for every $0 \neq \mathrm{a} \in \mathrm{R}$, there exists a positive integer $n$ such that $a^{n} \neq 0$ and any right $R$-homomorphism $f: a^{n} R \rightarrow M$ can be extended to $\mathrm{R} \rightarrow \mathrm{M}$. A ring $\mathrm{R}$ is called right $\mathrm{YJ}$-injective if $\mathrm{R}$ is $\mathrm{YJ}$-injective as a right $\mathrm{R}$-module. This notion was firstly introduced by [ 8 ]. A ring $\mathrm{R}$ is (Von Neumann) regular provided that for every $a \in R$ there exists $b \in R$ such that $a=a b a[7]$. $R$ is called a strongly regular ring if for each $a \in R, a \in a^{2} R$. In [9], proved that a ring $R$ is Von Neumann regular if and only if every cyclic right R-module is YJ-injective. So Von Neumann regular rings are right YJ-injective rings, but the converse is not true in general. Following [3], call $\mathrm{R}$ abelian if every idempotent element of $\mathrm{R}$ is central. A ring $\mathrm{R}$ is said to be reversible if $b a=0$ implies $a b=0$ for $a, b \in R$. A ring $R$ is called reduced if contains no non-zero nilpotent elements. A ring $R$ is said to be 2-primal [ 2 ] if $N(R)=P(R)$, where $P(R)$ is the prime radical of $\mathrm{R}$. 


\section{Completely YJ-injective :}

Following [ 5 ], a ring $\mathrm{R}$ is called $\pi$-regular ( strongly $\pi$-regular ) if for every element $a \in R$, there exists a positive integer $n$ and $b \in R$ such that $a^{n}=a^{n} b a^{n}\left(a^{n+1} b\right)$. We begin with the following definition.

\section{Definition 2.1:}

A ring $\mathrm{R}$ is called completely right ( left ) YJ-injective (briefly, right ( left ) CYJ-injective ) if every homomorphic image of $\mathrm{R}$ is right (left) $\mathrm{YJ}$-injective.

Obviously right ( left ) CYJ-injective rings are right ( left ) YJ-injective, but the converse is not true by Example ( 1 ).

\section{Proposition 2.2:}

A ring $\mathrm{R}$ is right $\mathrm{CYJ}$-injective if and only if every factor ring $\mathrm{R} / \mathrm{I}$ is a right $\mathrm{YJ}$ injective rings, where $\mathrm{I}$ is a two-sided ideal of $\mathrm{R}$.

\section{Proof :}

Given an ideal I of $R$, the natural map $\pi: R \rightarrow R / I$ define by $f(r)=r+I$, is an epimorphism since if $\mathrm{R}$ is CYJ-injective, then the homomorphic image $\mathrm{R} / \mathrm{I}$ is right $\mathrm{YJ}$ injective by Definition 2.1.

Conversely, assume that every factor ring R / I is a right $\mathrm{YJ}$-injective ring, where $\mathrm{I}$ is a two-sided ideal of $\mathrm{R}$. Then the argument above, every homomorphic image of $\mathrm{R}$ is right YJ-injective, concluding that $\mathrm{R}$ is right CYJ-injective.

\section{Example ( 1 ):}

Let $Z_{2}$ be the ring of integers modulo 2. Let $A=\left\{<a_{i}>/ a_{i} \in Z_{2}\right.$ and $a_{i}$ is eventually a constant $\}$. Then $A$ is a commutative Von Neumann regular ring. If $k \in Z_{2}$ and $<a_{1}, a_{2}, \ldots, a_{n}, a, a, a, \ldots>\in A$, let $k .<a_{1}, a_{2}, \ldots, a_{n}, a, a, a, \ldots>=k a$, then $Z_{2}$ is a right A-module. Consider a ring $R=\left[\begin{array}{cc}Z_{2} & Z_{2} \\ 0 & A\end{array}\right]$, then by [ 4, example 1$] \mathrm{R}$ is aright YJ-injective. We claim that $\mathrm{R}$ is not right CYJ-injective. Let $\mathrm{B}=\left\{\left\langle\mathrm{a}_{\mathrm{i}}\right\rangle \in \mathrm{A} / \mathrm{a}_{\mathrm{i}}\right.$ is eventually zero $\}$. Notice that $B$ is a proper two-sided ideal of A. Let $I=\left[\begin{array}{ll}0 & 0 \\ 0 & B\end{array}\right]$. Then I is a two sided ideal of $\mathrm{R}$. Consider a factor ring $\mathrm{R} / \mathrm{I}$. Then $R / I \cong\left[\begin{array}{cc}z_{2} & z_{2} \\ 0 & z_{2}\end{array}\right]$ as ring. However, $\left[\begin{array}{cc}z_{2} & z_{2} \\ 0 & z_{2}\end{array}\right]$ is not right YJ-injective. Hence a factor ring R / I is not right YJinjective and therefore $\mathrm{R}$ is not right $\mathrm{CYJ}$-injective.

\section{Remark ( 1 ) :}

Regular rings are right CYJ-injective but the converse is not true by the following example.

\section{Example ( 2 ) :}

Let $R=Z_{q^{2}}$, where $Z_{q^{2}}$ is the ring of integers modulo $q^{2}$ and $q$ is a prime number. Then $\mathrm{R}$ is a commutative CYJ-injective ring but it is not Von Neumann regular. 
Lemma 2.3 : [ 2 ]

A ring $R$ is 2-primal if and only if $R / P(R)$ is reduced

Lemma 2.4 : [ 1 ]

$\mathrm{R}$ is strongly regular ring if and only if $\mathrm{R}$ is reduced $\mathrm{YJ}$-injective ring.

Lemma 2.5 : [ 4 ]

For a ring $\mathrm{R}$, the following statements are equivalent:

( 1 ) $\mathrm{R}$ is strongly $\pi$-regular.

( 2 ) Each prime factor ring of $\mathrm{R}$ is strongly $\pi$-regular.

So we have the following

\section{Proposition 2.6 :} regular.

Let $\mathrm{R}$ be a 2-primal ring. If $\mathrm{R}$ is right CYJ-injective, then $\mathrm{R}$ is strongly $\pi$ -

Proof:

Since R is 2-primal, R / P( R ) is reduced by Lemma 2.3 and by Lemma 2.4, R / $\mathrm{P}(\mathrm{R})$ is strongly regular, so every prime factor ring of $\mathrm{R}$ is strongly $\pi$-regular. Therefore $\mathrm{R}$ is strongly $\pi$-regular by Lemma 2.5 .

Following [ 1 ], a ring $\mathrm{R}$ is called quasi-strongly right bounded ( briefly QSRB ) if every non zero maximal right ideal contains a non zero two sided ideal.

\section{Lemma 2.7: [ 1]}

Let $\mathrm{R}$ be a QSRB-ring. Then $\mathrm{R} / \mathrm{Y}(\mathrm{R})$ is reduced ring.

Lemma 2.8: [ 9]

If $R$ is a right $Y J$-injective, then $J(R)=Y(R)$.

\section{Theorem 2.9 :}

Let $\mathrm{R}$ be abelian QSRB-ring with nil $\mathrm{J}(\mathrm{R})$. If $\mathrm{R}$ is a CYJ-injective, then $\mathrm{R}$ is strongly $\pi$-regular ring.

\section{Proof :}

Since R is QSRB. Then by Lemma 2.7 R / Y ( R ) is reduced ring. And R / J( R ) is regular Lemmas (2.4and 2.8 ). Hence for any $x \in R$, there exists $y \in R$ such that $x-$ $x y x \in J(R)$. Write $x+J(R)=\bar{x}$. since $J(R)$ is nil, implies that there exists $e^{2}=e \in R$ such that $\overline{\mathrm{e}}=\bar{x} \bar{y}$ and $\overline{\mathrm{x}}=e \bar{x}$.

But $\mathrm{x}$-ex is nilpotent so there exists a positive integer $\mathrm{n}$ such that $(\mathrm{x}-\mathrm{ex})^{\mathrm{n}}=0$. Thus $\mathrm{x}^{\mathrm{n}} \in \mathrm{eR}$ because e is central i.e. $\mathrm{x}^{\mathrm{n}} \subseteq \mathrm{eR}$.

Next since $\overline{\mathrm{e}}=\bar{x} \bar{y}$ is central in $\mathrm{R} / \mathrm{J}(\mathrm{R})$ we have $\overline{\mathrm{e}}=\bar{x} \bar{y}=\bar{x} \bar{y} \bar{x} \bar{y}=\bar{x}^{2} \bar{y}^{2}=\ldots=\bar{x}^{n-n} \bar{y}^{n}$. Thus $\mathrm{e}-\mathrm{x}^{\mathrm{n}} \mathrm{y}^{\mathrm{n}} \in \mathrm{J}(\mathrm{R})$, and so $\left(\mathrm{e}-\mathrm{x}^{\mathrm{n}} \mathrm{y}^{\mathrm{n}}\right)^{\mathrm{m}}=0$ for some positive integer $\mathrm{m}$ because $\mathrm{J}(\mathrm{R})$ is nil. Consequently e $\in \mathrm{x}^{\mathrm{n}} \mathrm{R}$ i.e. $e \mathrm{R} \subseteq \mathrm{x}^{\mathrm{n}} \mathrm{R}$; hence we obtain $\mathrm{x}^{\mathrm{n}} \mathrm{R}=\mathrm{eR}$. But $\mathrm{eR}$ is a two-sided ideal in $\mathrm{R}$ and therefore $\mathrm{R}$ is strongly $\pi$-regular ring.

\section{Strongly CYJ-Injective Rings:}

In this section we investigate the Von Neumann regularity of rings $\mathrm{R}$ whose homomorphic images are YJ-injective as right R-modules. 


\section{Definition 3.1 :}

A ring $\mathrm{R}$ is called strongly right $\mathrm{CYJ}$-injective if every ring homomorphic image of $\mathrm{R}$ is $\mathrm{YJ}$-injective as a right $\mathrm{R}$-module.

\section{Proposition 3.2 :}

If $\mathrm{R}$ is a strongly right $\mathrm{CYJ}$-injective ring, then $\mathrm{R}$ is right $\mathrm{CYJ}$-injective.

\section{Proof :}

Let $\bar{R}=R / I$, where $\mathrm{I}$ is two sided ideal of $\mathrm{R}$. Since $\bar{R}$ is a YJ-injective right $\mathrm{R}$ module and for any $\mathrm{a} \in \mathrm{R}$, then there exists a positive integer $\mathrm{n}$ and $\bar{c} \in \bar{R}$ such that $\mathrm{f}\left(\mathrm{a}^{\mathrm{n}} \mathrm{b}\right)=\bar{c} a^{n} b$ for all $\mathrm{b} \in \mathrm{R}$. Now, let $\bar{f}$ be any non-zero $\bar{R}$-homomorphism $\bar{f}: \bar{a}^{n} \bar{R} \rightarrow \bar{R}$, we claim that, there exists $\bar{c} \in \bar{R}$ such that $f\left(\bar{a}^{n} \bar{b}\right)=\bar{c} \bar{a}^{n} \bar{b}$ for all $\bar{b} \in \bar{R}$. Let $\pi: a^{n} R \rightarrow \bar{a}^{n} \bar{R}$ denote the natural map. Then $f=\bar{f} o \pi: a^{n} R \rightarrow \bar{R}$. Since $\mathrm{f}\left(\mathrm{a}^{\mathrm{n}} \mathrm{b}\right)=\bar{c} \mathrm{a}^{\mathrm{n}} \mathrm{b}$ for all $\mathrm{b} \in \mathrm{R}$. Thus $\overline{\mathrm{f}}\left(\mathrm{a}^{\mathrm{n}} \overline{\mathrm{b}}\right)=\overline{\mathrm{f}} \mathrm{o} \pi\left(\mathrm{a}^{\mathrm{n}} \mathrm{b}\right)=\mathrm{f}\left(\mathrm{a}^{\mathrm{n}} \mathrm{b}\right)=\overline{\mathrm{ca}}{ }^{\mathrm{n}} \mathrm{b}=\overline{\mathrm{ca}}{ }^{\mathrm{n}} \mathrm{b}=\overline{\mathrm{ca}}{ }^{-\mathrm{n}} \overline{\mathrm{b}}$. So $\bar{R}$ is YJ-injective as a right $\bar{R}$-module. Therefore $\mathrm{R}$ is right CYJ-injective.

Recall that a ring $R$ is called ZI-ring if for every $a, b \in R, a b=0$ implies $a R b=0$. Clearly if $\mathrm{R}$ is ZI-ring then any right annihilator of $\mathrm{R}$ is two sided ideal [ 6 ].

\section{Theorem 3.3 :}

Let $\mathrm{R}$ be a strongly $\mathrm{CYJ}$-injective ring, then the following conditions are equivalent:
( 1 ) $\mathrm{R}$ is reduced.
( 2 ) $\mathrm{R}$ is reversible.
( 3 ) $\mathrm{R}$ is a ZI-ring.

\section{Proof :}

Obviously ( 1$) \Rightarrow(2) \Rightarrow$ ( 3 )

( 3 ) $\Rightarrow(1)$ Let $0 \neq a \in R$ such that $a^{2}=0$. Since $R$ is ZI-ring then $r(a)$ is two-sided ideal of R. Since R is CYJ-injective, and so any R-homomorphism of aR into R / r( a ) extends to one of $R$ into $R / r(a)$. Let $f: a R \rightarrow R / r(a)$ be defined by $f(a x)=x+r(a)$ for all $x \in R$. Then $f$ is well-defined. Since $R / r(a)$ is $Y J$-injective, there exists $c \in R$ such that $f(a x)=(c+r(a))$ ax $=c a x+r($ a $)$. Hence $f(a)=1+r(a)=c a+r(a)$. Therefore 1-ca $\in \mathrm{r}(\mathrm{a})$. But ca $\in \mathrm{r}(\mathrm{a})$ which implies $1 \in \mathrm{r}(\mathrm{a})$. So that $\mathrm{R}$ must be reduced. regular ring.

The following gives conditions on strongly right CYJ-injective to be strongly

\section{Theorem 3.4 :}

Let $\mathrm{R}$ be a ZI-ring. Then the following conditions are equivalent:

( 1 ) $\mathrm{R}$ is strongly regular ring

( 2 ) R is strongly CYJ-injective ring.

\section{Proof :}

( 1$) \Rightarrow(2)$ It is clear.

(2) $\Rightarrow(1)$ for all $a \in R, r(a)$ is two-sided. Therefore $R / r(a)$ is YJ-injective and so there exists a positive integer $n$ such that $a^{n} \neq 0$ and any $R$-homomorphism of $a^{n} R$ into $R / r(a)$ extended to one of $\mathrm{R}$ into $\mathrm{R} / \mathrm{r}(\mathrm{a})$. 
Let $\mathrm{f}: \mathrm{a}^{\mathrm{n}} \mathrm{R} \rightarrow \mathrm{R} / \mathrm{r}(\mathrm{a})$ be defined by $\mathrm{f}\left(\mathrm{a}^{\mathrm{n}} \mathrm{r}\right)=\mathrm{r}+\mathrm{r}(\mathrm{a})$, then $\mathrm{f}$ is well-defined $\mathrm{R}$ homomorphism. Indeed if $a^{n} r_{1}=a^{n} r_{2}$, then $r_{1}-r_{2} \in r\left(a^{n}\right)$. Since R ZI-ring, then by Theorem 3.3 $\mathrm{R}$ is reduced. Hence $\mathrm{r}\left(\mathrm{a}^{\mathrm{n}}\right)=\mathrm{r}(\mathrm{a})$. Therefore $r_{1}+r(\mathrm{a})=\mathrm{r}_{2}+\mathrm{r}(\mathrm{a})$. Since $\mathrm{R} / \mathrm{r}(\mathrm{a})$ is YJ-injective, there exists $\mathrm{c} \in \mathrm{R}$ such that $\mathrm{f}\left(\mathrm{a}^{\mathrm{n}} \mathrm{r}\right)=(\mathrm{c}+\mathrm{r}(\mathrm{a}))\left(\mathrm{a}^{\mathrm{n}} \mathrm{r}\right)=\mathrm{ca}^{\mathrm{n}} \mathrm{r}+$ $r(a)$ so that $f\left(a^{n}\right)=1+r(a)=c a^{n}+r(a)$ which implies that $1-c a^{n} \in r(a)=1($ a $)$. Hence $\mathrm{a}=\mathrm{ba}^{2}$, where $\mathrm{b}=\mathrm{ca}^{\mathrm{n}-1}$. So that $\mathrm{R}$ is strongly regular rings.

Recall that a ring $\mathrm{R}$ is MERT [ 6 ], if every maximal essential right ideal of $\mathrm{R}$ is a two sided ideal of $\mathrm{R}$. Now, the following result is given.

\section{Theorem 3.5}

Let $\mathrm{R}$ be MERT strongly right CYJ-injective ring, then $\mathrm{Y}(\mathrm{R})=0$.

\section{Proof :}

If $Y(R) \neq 0$, then there exists $0 \neq x \in R$ such that $x^{2}=0$. Since $r(x) \neq R$, there exists a maximal right ideal $M$ of $R$ such that $r(x) \subseteq M$. Thus $M$ is an essential right ideal and so $M$ is two-sided ideal. Let $f: x R \rightarrow R / M$ defined by $f(x r)=r+M$. Note that $\mathrm{f}$ is well-defined R-homomorphism. Since $\mathrm{R} / \mathrm{M}$ is $\mathrm{YJ}$-injective, there exists $\mathrm{y} \in \mathrm{R}$ such that $1+M=f(x)=y x+M$. Thus $1-y x \in M$, whence $1 \in M$, which is a contradiction. Therefore $\mathrm{Y}(\mathrm{R})=0$.

\section{Theorem 3.6 :}

Let $\mathrm{R}$ be a MERT-ring. Then the following conditions are equivalents:

( 1 ) $\mathrm{R}$ is strongly regular ring.

( 2 ) $\mathrm{R}$ is a strongly right CYJ-injective.

\section{Proof :}

By Theorem3.5, $Y(R)=0$, since $R$ is $Y J$-injective then $J(R)=Y(R)=0$. Therefore $R$ is semi-prime. We have to prove that $R$ is reduced. Let $0 \neq a \in R$ such that $a^{2}=0$. Then there exists a maximal right ideal $M$ of $R$ containing $r(a)$. Since $R$ is a semi-prime, then $M$ must be essential right ideal and so $M$ is a two-sided. By similar methods used in Theorem 3.5 we have $1 \in \mathrm{M}$, which is a contradiction, so that $\mathrm{R}$ is reduced and by Theorem 3.4 we have $\mathrm{R}$ is a strongly regular ring.

\section{Theorem 3.7 :}

The following conditions are equivalent for any ring $\mathrm{R}$ :

( 1 ) $\mathrm{R}$ is strongly regular ring.

( 2 ) $\mathrm{R}$ is SLB and strongly right CYJ-injective ring.

\section{Proof :}

$(1) \Rightarrow(2)$ It is clear.

( 2 ) $\Rightarrow$ ( 1 ) We will show that $R$ is reduced. Suppose that $a^{2}=0$ with $a \neq 0$. Then $\operatorname{lr}(a)$ is is non-zero left ideal of $\mathrm{R}$. Thus there exists a non-zero two-sided ideal I of $\mathrm{R}$ such that $\mathrm{I} \subseteq \operatorname{lr}(\mathrm{a})$ and so $\mathrm{r}(\mathrm{a}) \subseteq \mathrm{r}(\mathrm{I})$.

Now, $\mathrm{R} / \mathrm{r}$ ( I ) is YJ-injective right R-module. Let $\mathrm{f}: \mathrm{aR} \rightarrow \mathrm{R} / \mathrm{r}$ ( I ) be defined by $\mathrm{f}($ ar $)=r+r(I)$. Then $\mathrm{f}$ is a well-defined right $\mathrm{R}$-homomorphism. Since $\mathrm{R} / \mathrm{r}(\mathrm{I})$ is YJ-injective right R-module, there exists $c \in R$ such that $1+r(I)=f(a)=c a+r(I)$. Thus $1-c a \in r(I)$; whence $1 \in \mathrm{r}(\mathrm{I})$, which is a contradiction. Therefore $\mathrm{R}$ is reduced and hence $\mathrm{R}$ is strongly regular. 


\section{REFERENCES}

[1] Abdullah, J. S. A, On YJ-Injectivity, PH. D., Thesis University Of Mosul-Iraq (2003).

[2] Birkenmeier, G. F., Kim J. Y. and Park J. K. (1994 ), A Connection between Weak Regularity And The Simplicity of Prime Factor Rings, American Math. Soc. 122( 1 ) pp. 53-58.

[3] Chen, J. and Ding N. (1999), On General Principally Injective Rings. Comm. In Algebra 27 (5) pp. 2097-2116.

[4] Chen, J. And Ding, N. ( 2001 ), On Regularity On Rings. Algebra Colloq. 8, pp. 267-274.

[5] Fisher, J. W. and Snider, R. L. ( 1974 ), On the Von Neumann Regularity of Rings with Regular Prime Factor Rings. Pacific J. Math. 54 (1) , pp. 135-144.

[6] Kim, N. K. And Kim, J. Y. ( 1999 ), On Simple Singular GP-Injective Module, Communications In Algebra 27 ( 5 ), pp. 2087-2096.

[7] Ming, R. Y. C. (1980), On V-Rings and Prime Rings, J. Algebra, 62 pp. 13-20.

[8] Ming, R. Y. C. ( 1986 ), On Von Neumann Regular Rings XI, Bull. Math. Soc., 30 (76), pp.371-379.

[9] Nam, S. B., Kim N. K. And Kim, J. Y. ( 1995 ), On Simple GP-Injective Module, Communications In Algebra 23 ( 14 ), pp. 5437-5444. 